\title{
Hypertension Undermines Mineralization-inducing Capacity of and Tissue Response to Mineral Trioxide Aggregate Endodontic Cement
}

\author{
Christine Men Martins, DDS, MSc, João Eduardo Gomes-Filbo, DDS, MSc, PhD, \\ Índia Olinta de Azevedo Queiroz, DDS, MSc, Edilson Ervolino, DDS, MSc, PhD, \\ and Luciano Tavares Angelo Cintra, DDS, MSc, PbD
}

\begin{abstract}
Ahstract
Introduction: This study evaluated the effect of hypertension on tissue response to and mineralization capacity of white and gray mineral trioxide aggregate (MTA) (Angelus Industry Ontological Products, Londrina, Brazil), an endodontic reparative cement. Methods: Polyethylene tubes containing gray MTA, white MTA, or intermediate restorative material (positive control) or an empty tube (negative control) were implanted into the dorsal connective tissue of spontaneous hypertensive and Wistar rats ( $n=12$ each). Six rats in each group were sacrificed after 7 days, and the remainder after 30 days. Tubes with surrounding tissue were removed, and a histologic analysis was performed using hematoxylin-eosin and von Kossa staining and examination by polarized light microscopy. Results: The inflammatory response to all materials was greater in hypertensive compared with normotensive rats $(P<.05)$. Positive von Kossa staining and birefringent structures in polarized light were observed for both gray and white MTA $(P>.05)$, but these were more pronounced in normotensive rats $(P<.05)$. Necrotic areas with positive von Kossa staining were observed for intermediate restorative material. Conclusions: Hypertension undermines tissue repair and mineralization, which can negatively affect treatment outcome. Nonetheless, mineralization in response to MTA was observed even under hypertensive conditions. (J Endod 2016;42:604-609)
\end{abstract}

\section{Key Words}

Calcification, hypertension, periapical diseases

From the Restorative Dentistry Department, Endodontic Area, Aracatuba Dental School, Universidade Estadual Paulista, Aracatuba, São Paulo, Brazil.

Address requests for reprints to Prof João Eduardo GomesFilho, Restorative Dentistry Department, Endodontic Area, Aracatuba Dental School, Universidade Estadual Paulista, Rua José Bonifácio, 1193, 16015-050, Aracatuba, São Paulo, Brazil. E-mail address: joao@foa.unesp.br

0099-2399/\$ - see front matter

Copyright (C) 2016 American Association of Endodontists. http://dx.doi.org/10.1016/j.joen.2016.01.003
Wypertension is a disease that affects around 25\% of the general population and 50\% 7 of individuals over 60 years old (1). It is defined as high blood pressure (ie, systolic blood pressure $>140 \mathrm{~mm} \mathrm{Hg}$, diastolic blood pressure $>90 \mathrm{~mm} \mathrm{Hg}$, or both) (1).

Hypertension is considered an inflammatory disease that has systemic effects, including calcium loss from the body $(2,3)$. It has been suggested that hypertension is an inflammatory vascular process that increases $\mathrm{T}$ lymphocyte and natural killer cell infiltration and proliferation, with deposition of perivascular macrophages and increased expression of collagen type I and III, interleukin 16, lymphocyte chemotactic factor, and cytotoxic T-lymphocyte-associated protein 4 (2-5). Moreover, it is associated with an increase in C-reactive protein, which is considered an inflammatory marker (6).

Epidemiological studies have found an association between periodontal disease and hypertension that implicates oral infection and the immune/inflammatory response (7). It was observed that $24.6 \%$ of patients hospitalized for periapical abscesses had hypertension (8). Periapical lesions occur as an inflammatory response to infection; along with hypertension, it can lead to vascular injury and inflammation. However, there is no study linking hypertension and periapical lesion healing in the literature.

Hypertension can also lead to abnormalities in mineralization because of increased serum levels of the parathyroid hormone, whose main function is to stimulate the breakdown of bone by activation and proliferation of osteoclasts and thereby increase blood calcium levels. Abnormal vitamin D metabolism causes reduced serum ionized calcium and decreases calcium absorption (3). In addition, trabecular bone mineral content is decreased in both children and adults $(9,10)$, which is observed not only in pre-existing but also in newly formed medullar bone tissue (11). This morbidity leads to defects in bone healing $(9,11,12)$ and a high rate of implant loss because of defects in osseointegration $(12,13)$. Hypertension may also increase the receptor activator of nuclear factor $\kappa \mathrm{B}$ ligand/osteoprotegerin ratio, which would tend to favor a more osteoclastic state (9). Thus, hypertension can affect bone metabolism and may be a risk factor for bone diseases including periodontitis, periapical lesions, and rheumatoid arthritis (14).

Endodontic treatment aims to control infection and restore apical and periapical tissues (15). Apical periodontitis may persist because of the presence of bacteria in the apical root canal space (16), which can be treated by endodontic surgery with retrofilling (16). The endodontic reparative cement mineral trioxide aggregate (MTA) is used in this process because of its excellent physical, chemical, and biological properties, including its ability to induce mineralization in tissues at the site of application (17-19).

Few studies have investigated the difference between the white and gray types of MTA with respect to their potential for inducing tissue regeneration, biocompatibility, and sealing ability (20-22). In the present study, we investigated whether hypertension affects the tissue response to white and gray MTA implanted subcutaneously into rats as well as the ability of these substances to induce mineralization. 


\section{Methods}

The study was approved by the Ethical Committee of Aracatuba Dental School, Aracatuba, São Paulo, Brazil (protocol no. 201300961).

\section{Animals}

Male spontaneously hypertensive and Wistar rats $(n=12$ each, age: 2-3 months, 250-280 g) were used as hypertensive (experimental) and normotensive (control) animals, respectively.

\section{Surgical Procedures}

White and gray MTA (Angelus Industry Ontological Products, Londrina, Brazil) and intermediate restorative material (IRM) (Dentsply Caulk, Milford, DE) were prepared according to the manufacturers' recommendations and placed in 72 sterile polyethylene tubes (internal diameter $=1.0 \mathrm{~mm}$, external diameter $=1.6 \mathrm{~mm}$, length $=10 \mathrm{~mm}$ ) (Abbott Labs of Brazil, São Paulo, Brazil) with a Lentulo spiral (Maillefer Dentsply, Tulsa, OK); 24 empty tubes were used as the control. Each tube was completely filled with the material.

Animals were anesthetized with xylazine $(10 \mathrm{mg} / \mathrm{kg}$ ) (Anasedan/Vetbrands Division Animal Health, São Paulo, Brazil) and ketamine (25 mg/ kg) (Cetamin Syntec do Brazil, São Paulo, Brazil). Their backs were shaved and the skin disinfected with 5\% iodine solution; a 2-cm incision was made from head to tail with a \#15 Bard-Parker blade (Becton-Dickinson, Franklin Lakes, NJ). Four pockets $6 \mathrm{~cm}$ apart were created on each side of the incision into which 3 tubes (filled with white or gray MTA or IRM) and an empty tube were implanted. The skin was closed using Ethicon 4.0 silk sutures (Johnson \& Johnson, Sao Paulo, Brazil).

\section{Histologic Analysis}

On 7 and 30 days after tube implantation, animals were euthanized by an overdose of anesthetic, and the tubes with surrounding tissue were removed and fixed in $10 \%$ formalin solution $(\mathrm{pH}=7.0)$ and cut transversely into 2 halves that were each cut longitudinally using a sharp blade to allow contact between the surfaces and processing solutions. Specimens were embedded in glycol methacrylate with histore$\sin$. Serial sections $3-\mu \mathrm{m}$ thick were stained with hematoxylin-eosin, whereas $10-\mu \mathrm{m}$ sections were processed by von Kossa staining or directly examined under polarized light (23).

Tissue in contact with the material at the tube opening was assessed by counting the average number of inflammatory cells stained by hematoxylin-eosin in 10 randomly selected microscopic fields at $400 \times$ magnification by 1 evaluator blinded to the experimental condition. Mineralization per $\mu \mathrm{m}^{2}$ was determined using Qwin software (Leica Microsystems, Wetzlar, Germany). An average value for each material was determined as the total number of cells counted in 10 microscopic fields at $400 \times$ magnification. Results were evaluated by 3 -way analysis of variance, and $P$ values $<.05$ were considered significant.

\section{Results \\ Hypertension Exacerbates the Inflammatory Response to Endodontic Reparative Cement}

The average number of inflammatory cells at the mouth of polyethylene tubes was determined on days 7 and 30 (Table 1). The inflammatory response to gray MTA decreased over time and was similar to the response to white MTA under normotensive conditions. However, the inflammatory response was increased in hypertensive rats $(P<.05)$ and also in the presence of IRM relative to the control group. All materials induced greater inflammation in hypertensive compared with normotensive rats $(P<.05)$.

The inflammatory response to all materials as well as to the empty tube was exacerbated by hypertension (Fig. 1A1-D4). Macrophages and lymphocytes were the predominant cell types detected in the tissue. Fibrous capsules were observed surrounding the tubes under both hyper- and normotensive conditions that were thinner at 30 versus 7 days.

TABLE 1. Inflammatory Cells and Mineralization Area Average in Hypertension and Normotension Conditions

\begin{tabular}{|c|c|c|c|c|}
\hline \multirow[b]{2}{*}{ Material } & \multicolumn{2}{|c|}{7 days } & \multicolumn{2}{|c|}{30 days } \\
\hline & $\begin{array}{c}\text { Normotensive } \\
\text { Mean } \pm \text { SD }\end{array}$ & $\begin{array}{l}\text { Hypertensive } \\
\text { Mean } \pm \text { SD }\end{array}$ & $\begin{array}{c}\text { Normotensive } \\
\text { Mean } \pm \text { SD }\end{array}$ & $\begin{array}{l}\text { Hypertensive } \\
\text { Mean } \pm \text { SD }\end{array}$ \\
\hline \multicolumn{5}{|c|}{ Inflammatory-hematoxylin eosin* } \\
\hline MTA gray & $235.00 \pm 96.29$ & $303.55 \pm 50.36^{\ddagger}$ & $194.18 \pm 25.41$ & $206.22 \pm 47.82^{\ddagger}$ \\
\hline MTA white & $215.38 \pm 30.69$ & $334.25 \pm 117.61^{\ddagger}$ & $179.08 \pm 66.16$ & $371.42 \pm 122.39^{\ddagger}$ \\
\hline IRM & $177.60 \pm 58.61$ & $213.20 \pm 39.00^{\ddagger}$ & $326.78 \pm 96.77$ & $374.00 \pm 56.15^{\ddagger}$ \\
\hline Control & $376.37 \pm 92.27$ & $301.34 \pm 95.27$ & $303.62 \pm 58.37$ & $297.63 \pm 63.23$ \\
\hline \multicolumn{5}{|c|}{ Mineralization-Von Kossa $^{\dagger}$} \\
\hline MTA gray & $395,447.41 \pm 121,643.92$ & $468,748.73 \pm 74,096.83^{\S}$ & $307,126.92 \pm 40,447.09$ & $315,192.98 \pm 43,582.08^{5}$ \\
\hline MTA white & $477,389.78 \pm 113,190.85$ & $288,507.07 \pm 44,017.33^{\S}$ & $570,670.05 \pm 8955.82$ & $336,474.21 \pm 29,138.79^{\S}$ \\
\hline IRM & $155,233.13 \pm 52,021.17$ & $291,139.31 \pm 51,986.91^{\S}$ & $63,600.92 \pm 25,962.82$ & $206,223.83 \pm 57,863.62^{\S}$ \\
\hline Control & $0.00 \pm 0$ & $0.00 \pm 0$ & $0,00 \pm 0$ & $0.00 \pm 0$ \\
\hline \multicolumn{5}{|c|}{ Mineralization-polarized light ${ }^{\dagger}$} \\
\hline MTA gray & $94,564.65 \pm 19,793.50$ & $69,745.84 \pm 12,573.40^{\S}$ & $22,018.27 \pm 5074.59$ & $65,392.96 \pm 6573.77^{\S}$ \\
\hline MTA white & $319,889.79 \pm 96,245.49$ & $216,619.38 \pm 37,825.46^{\S}$ & $48,069.21 \pm 11,328.04$ & $235,522.79 \pm 35,704.59^{\S}$ \\
\hline IRM & $0.00^{\text {ๆ }} \pm 0$ & $0.00 \pm 0^{\text {ๆ }}$ & $0.00 \pm 0^{q}$ & $0.00 \pm 0^{\pi}$ \\
\hline Control & $0.00 \pm 0$ & $0.00 \pm 0$ & $0.00 \pm 0$ & $0.00 \pm 0$ \\
\hline
\end{tabular}

IRM, intermediate restorative material; MTA, mineral trioxide aggregate; SD, standard deviation.

*The mean number of inflammatory cells ( $n=6$ per group).

${ }^{\dagger}$ The mean area of mineralization associated with white or gray MTA, IRM, or empty tubes at 7 and 30 days in hyper- and normotensive rats as determined by von Kossa staining and polarized light microscopy ( $n=6$ per group)

${ }^{\star}$ Significant difference between hypertensive and normotensive groups for white MTA, gray MTA, and IRM $(P<.05)$.

${ }^{\S}$ Significant difference between days 7 and 30 for gray and white MTA and IRM in the hypertensive versus normotensive group.

"Significant difference between the control and other materials

"Significant difference between IRM and both types of MTA. 


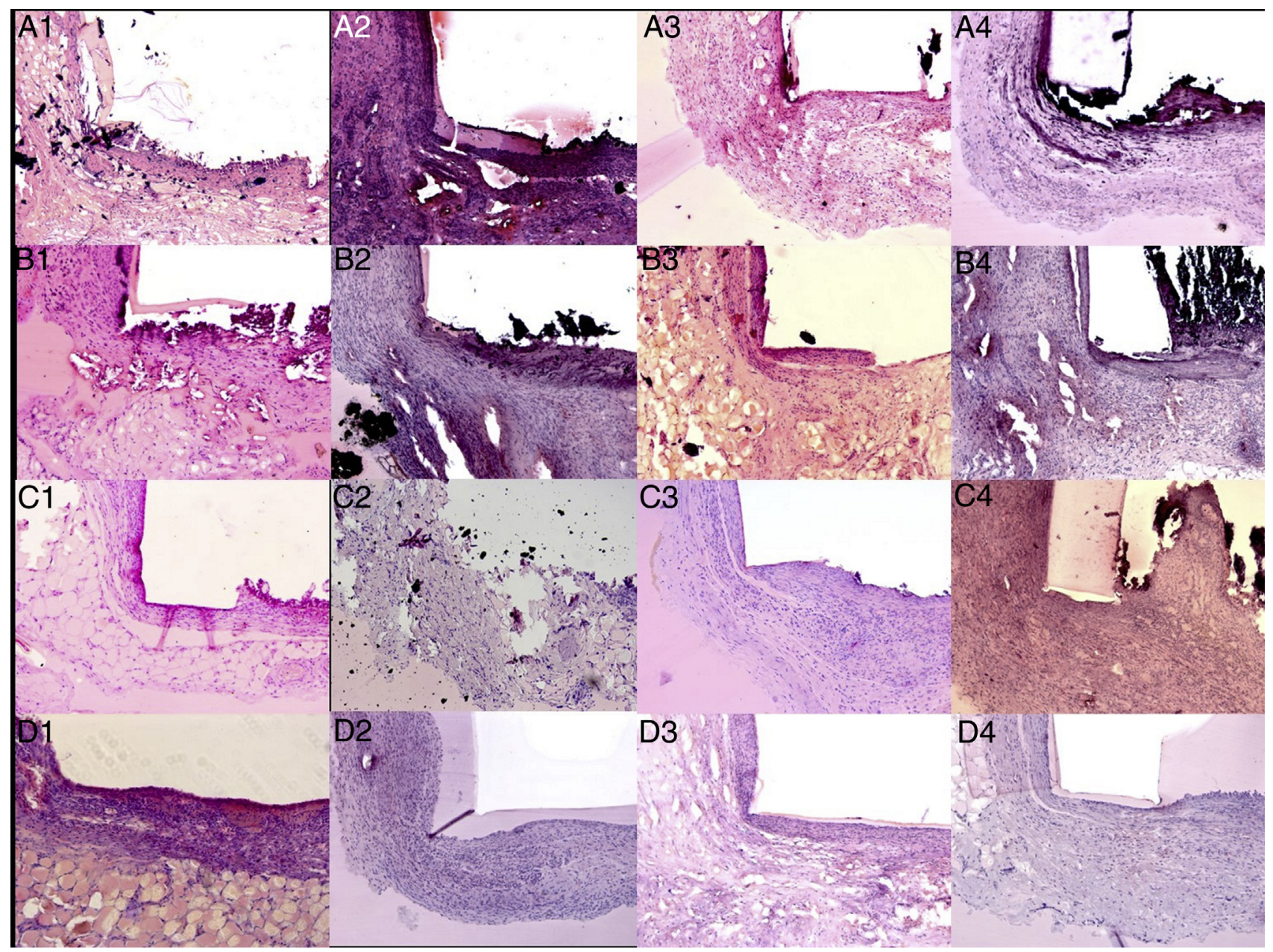

Figure 1. Inflammatory response to white or gray MTA, IRM, or empty tubes at 7 and 30 days in hyper- and normotensive rats as determined by hematoxylin-eosin staining ( $n=6$ per group). (A) Gray MTA, $(B)$ white MTA, $(C)$ IRM, and $(D)$ control. (1) Normotensive day 7, (2) hypertensive day 7, (3) normotensive day 30, and (4) hypertensive day 30 . Hematoxylin-eosin staining $(100 \times)$ shows fibrous capsule formation with infiltration of macrophages and lymphocytes. The inflammatory response was increased by hypertension and in the presence of MTA and IRM.

\section{Hypertension Inhibits Mineralization}

An analysis of birefringence under polarized light revealed the presence of calcium carbonate particles for white and gray MTA but not for the other 2groups (Table 1). Under normotensive conditions, the birefringent area $\left(\mu \mathrm{m}^{2}\right)$ was greater on day 30 than on day 7 $(P<.05)$; however, in hypertensive rats, the areas were similar at both time points. The mineralized area in contact with white MTA versus other substances was greater in both hyper- and normotensive rats; there were no birefringent areas observed for IRM or the control group $(P<.05)$.

Calcium particles were observed by von Kossa staining, with dark areas representing tissue necrosis. Under hypertensive conditions, mineralization was similar on day 30 for both types of MTA and higher than in the IRM group. A decrease in mineralization was observed over time for gray MTA $(P<.05)$; however, for white MTA, this trend was noted only in normotensive rats, whereas under hypertensive conditions mineralization was increased at 30 days compared with 7 days $(P<.05)$. The area of positive staining was lower overall in the IRM group in which hypertension exacerbated this response $(P<.05)$. There were no areas positive for von Kossa staining in the control group $(P<.05)$.

Based on von Kossa staining (Fig. 2A1-D4) and polarized light microscopic (Fig. 3A1-D4) analyses, the 2 types of MTA had more mineralization than the other groups. In general, mineralization was more consistently observed in the absence of hypertension.

\section{Discussion}

This is the first study evaluating the effect of hypertension on tissue response to MTA, including mineralization. The results show that hypertension enhances the inflammatory response and inhibits the capacity of MTA to induce mineralization.

Hypertension is an inflammatory vascular process (2) in which high levels of angiotensin II stimulate the infiltration and proliferation of T lymphocytes and natural killer cells (24). In addition, increases in aldosterone and sodium production enhance the accumulation of perivascular macrophages in the heart (4) and induce the expression of collagen type I and III, interleukin 16, lymphocyte chemotactic factor, and cytotoxic T-lymphocyte-associated protein 4 , which are associated with arterial inflammatory lesions, fibrosis, and vascular calcification (5). Hypertension is also associated with the up-regulation of C-reactive protein, an inflammatory marker that also promotes inflammation by stimulating the release of proinflammatory cytokines such as interleukin 6 , interleukin $1 \beta$, and tumor necrosis factor alpha by monocytes and induces the expression of intracellular and vascular cell adhesion molecules by endothelial cells (6). 
Basic Research-Biology

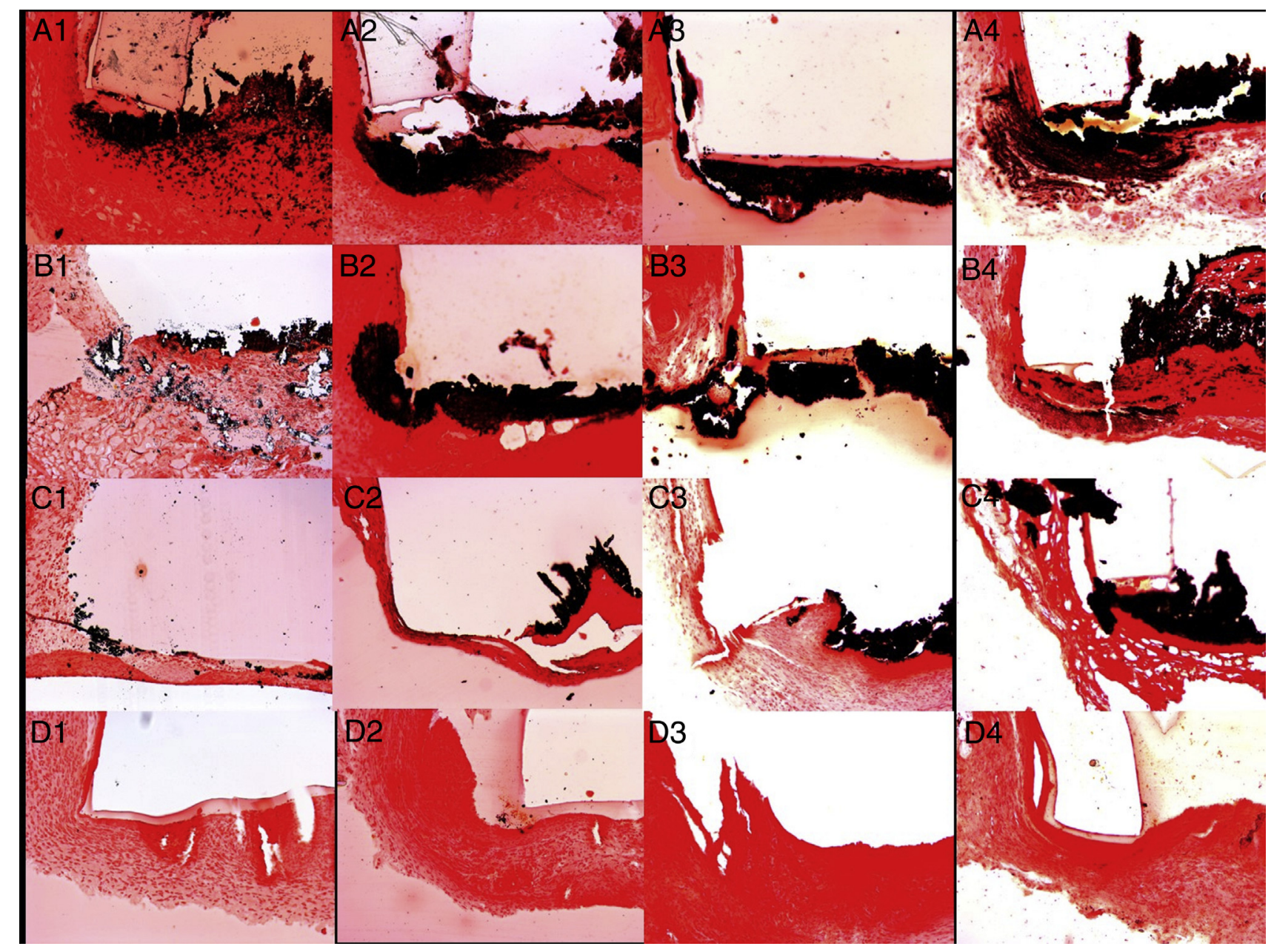

Figure 2. Mineralization in response to white or gray MTA, IRM, or empty tubes at 7 and 30 days in hyper- and normotensive rats as determined by von Kossa staining $(100 \times)$. (A) Gray MTA, $(B)$ white MTA, $(C)$ IRM, and $(D)$ control. (1) Normotensive day 7, (2) hypertensive day 7, (3) normotensive day 30, and (4) hypertensive day 30. The black areas represent mineralization. Both types of MTA induced mineralization, which was not observed for IRM or the control group; the dark areas of tissue associated with IRM were necrotic. More consistent mineralization was observed in normotensive rats, especially at 30 days.

In the present study, we observed that hypertension increased inflammation in response to a foreign substance. The combined effect of hypertension and endodontic cement increased inflammation as evidenced by the higher number of neutrophils in hypertensive compared with normotensive rats. This is consistent with another report showing that the effects of foreign materials on inflammation are exacerbated when there is coexisting hypertension (25).

Mineralization was evaluated by von Kossa staining and birefringence under polarized light. The former is used to detect calcium deposits and, hence, the mineralization area (26). On the other hand, polarized light reveals birefringent calcium carbonate particles in the tissue and as such provides insight into the mechanism by which mineralization occurs because calcium carbonate nuclei initiate mineralization (27).

Hypertension caused less consistent mineralization, possibly because of increased mobilization of calcium from bone and calcium excretion by the kidneys under these conditions (3). Secondary activation of the parathyroid hormone leads to the activation of vitamin D, which stimulates the breakdown of bone by activation and the proliferation of osteoclasts; calcium release is thereby increased and is absorbed by the intestine and resorbed by the kidneys (3) because of the high levels of angiotensin II (28-30).
Under normotensive conditions, gray MTA was associated with a smaller birefringent area at 30 days compared with 7 days. This was not surprising because in the days after surgery tissue is regenerating and has a larger birefringent area, which is more restricted during the healing period (31). Under hypertensive conditions, birefringence was maintained over time; this may be explained by the presence of exudate from the persistent inflammatory response, which caused ion solubilization and diffusion (32).

Greater birefringence was observed in response to white compared with gray MTA. This can be ascribed to the different properties of the 2 MTAs. Studies on leakage, tissue and osteoblast biocompatibility, pulp wound healing, and hard tissue bridge formation showed similar results for white and gray MTA $(22,33)$. In contrast, some studies have found that gray MTA promotes the growth, adherence, and differentiation of osteoblastic cells while showing poor leakage results (34). White MTA has a lower setting time than gray MTA, which explains its higher solubility and ionic release $(20,22-34)$. However, gray MTA has high levels of arsenic, tetracalcium aluminoferrite, iron, cadmium, and chromium, which can lead to rejection by tissue, inflammation, or allergic reactions and thereby compromise mineralization (20). Although these properties can induce greater calcium carbonate formation, we did not detect increased mineralization with this substance by von Kossa staining. 


\section{Basic Research-Biology}

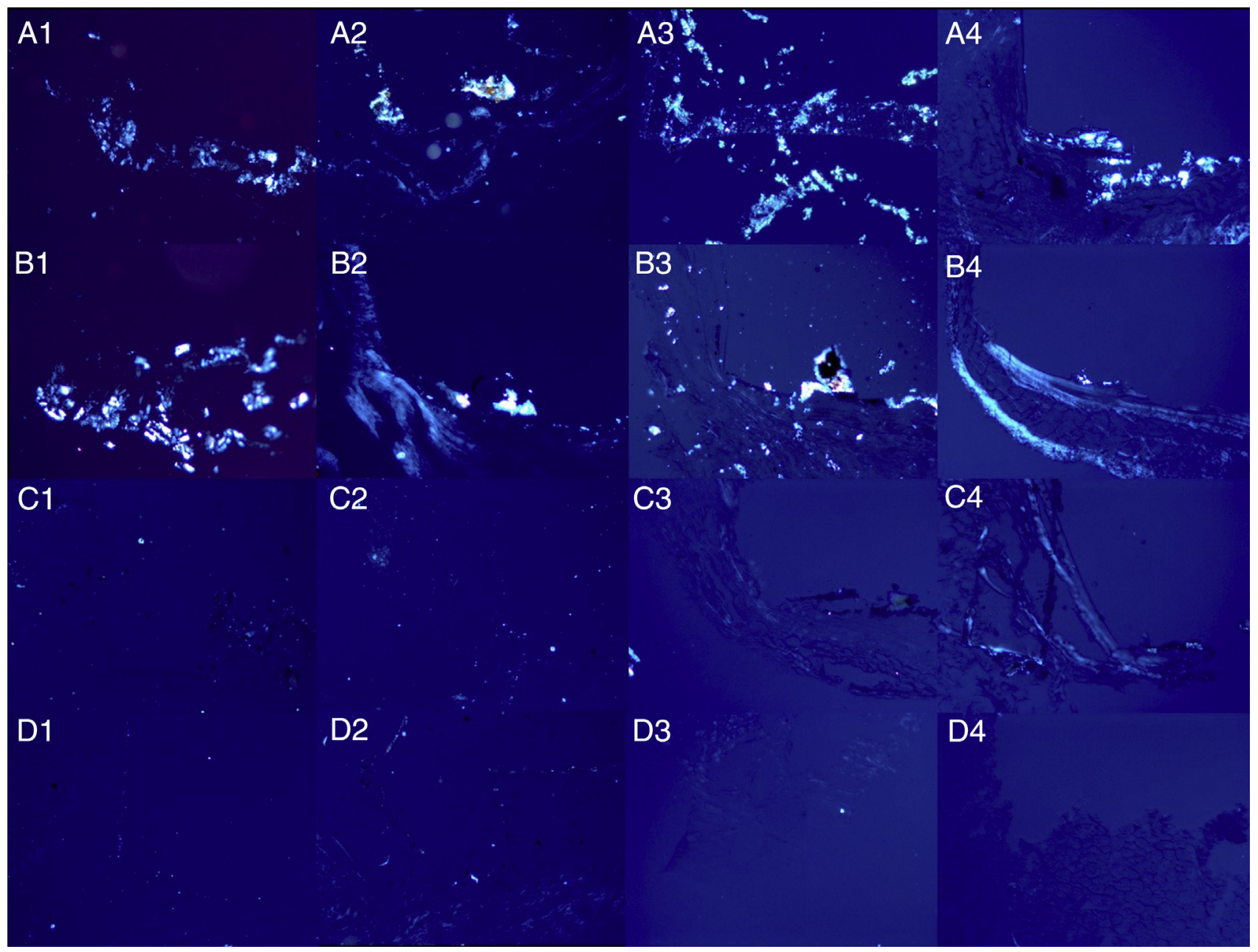

Figure 3. Mineralization in response to white or gray MTA, IRM, or empty tubes at 7 and 30 days in hyper- and normotensive rats as determined by polarized light microscopy $(100 \times)$. (A) Gray MTA, $(B)$ white MTA, $(C)$ IRM, and $(D)$ control. (1) Normotensive day $7,(2)$ hypertensive day $7,(3)$ normotensive day 30 , and $(4)$ hypertensive day 30. Birefringent structures in the tissue represent calcite crystals, which were observed for different types of MTA but not for IRM or the control group. More consistent birefringence was observed in normotensive rats, especially at 30 days.

Mineralization in response to MTA was observed even under hypertensive conditions. MTA contains calcium oxide and calcium phosphate; the former reacts with water from tissue to form calcium hydroxide, which dissociates into calcium and hydroxyl ions. Calcium ions react in turn with carbon dioxide in tissue to form crystals of calcite - a polymorph of calcium carbonate - that are birefringent under polarized light and are deposited in hard tissue (34). Necrotic areas may also be positive for von Kossa staining; this is consistent with the absence of polarization observed with IRM, which is toxic and causes necrosis upon direct contact with tissue but does not release calcium to form calcium carbonate.

MTA is biocompatible and exhibits good marginal sealing; in addition, it induces dentinogenesis, cementogenesis, and osteogenesis (18, 19). The hydration chemistry of MTA is important; it dictates its chemical stability, physical durability, biocompatibility, and activities in tissue. When MTA powder is mixed with water, its components alite and belite react to form a poorly crystalline calcium silicate hydrate gel and portlandite, whereas the aluminate, ferrite, and gypsum react with water to generate ettringite, which is converted into thermodynamically stable monosulfate (35).

In conclusion, although hypertension can jeopardize tissue repairincluding mineralization - and thus negatively impact endodontic treatment outcome, MTA still has satisfactory mineralization ability.

\section{Acknowledgments}

The authors deny any conflicts of interest related to this study.

\section{References}

1. World Health Organization. A Global Brief on Hypertension: Silent Killer, Global Public Health Crisis. Gevena: World Health Organization; 2013.

2. Marvar PJ, Thabet SR, Guzik TJ, et al. Central and peripheral mechanisms of T-lymphocyte activation and vascular inflammation produced by angiotensin H-induced hypertension. Circ Res 2010;107:263-70.

3. Izawa Y, Sagara K, Kadota T, Makita T. Bone disorders in spontaneously hypertensive rat. Calcif Tissue Int 1985;37:605-7.

4. Rocha R, Rudolph AE, Frierdich GE, et al. Aldosterone induces a vascular inflammatory phenotype in the rat heart. Am J Physiol Heart Circ Physiol 2002;283: H1802-10.

5. Sararoudi RB, Kheirabadi GR, Kousha M, et al. Is there any association of personality traits with vascular endothelial function or systemic inflammation? Adv Biomed Res 2014;3:210.

6. Dinh QN, Drummond GR, Sobey CG, Chrissobolis S. Roles of inflammation, oxidative stress, and vascular dysfunction in hypertension. Biomed Res Int 2014;2014: 406960

7. Tsioufis C, Kasiakogias A, Thomopoulos C, Stefanadis C. Periodontitis and blood pressure: the concept of dental hypertension. Atherosclerosis 2011;219:1-9.

8. Allareddy V, Lin CY, Shah A, et al. Outcomes in patients hospitalized for periapical abscess in the United States: an analysis involving the use of a nationwide inpatient sample. J Am Dent Assoc 2010;141:1107-16. 
9. Bastos MF, Brilhante FV, Bezerra JP, et al. Trabecular bone area and bone healing in spontaneously hypertensive rats. A histometric study. Braz Oral Res 2010; 24:170-6.

10. Inoue T, Moriya A, Goto K, et al. What is the difference of bone growth in SHR and SD rats? Clin Exp Pharmacol Physiol 2007;22:242-3.

11. Bastos MF, Brilhante FV, Gonçalves TE, et al. Hypertension may affect toothsuporting alveolar bone quality: a study in rats. J Periodontol 2010;81: $1075-83$.

12. Manrique N, Pereira CC, Garcia LM, et al. Alveolar bone healing process in spontaneously hypertensive rats (SHR), a radiographic densitometry study. J Appl Oral Sci 2012;20:222-7.

13. Alsaadi G, Quirynen M, Komárek A, van Steenberghe D. Impact of local and systemic factors on the incidence of late oral implant loss. Clin Oral Implants Res 2008;19: 670-6.

14. Panoulas VF, Metsios GS, Pace AV, et al. Hypertension in rheumatoid arthritis. Rheumatology (Oxford) 2008;47:1286-98.

15. Holland R, Otoboni Filho JA, de Souza V, et al. A comparison of one versus two appointment endodontic therapy in dogs' teeth with apical periodontitis. J Endod 2003;29:121-4.

16. Ray JJ, Kirkpatrick TC. Healing of apical periodontitis through modern endodontic retreatment techniques. Gen Dent 2013;61:19-23.

17. Felippe WT, Felippe MC, Rocha MJ. The effect of mineral trioxide aggregate on the apexification and periapical healing of teeth with incomplete root formation. J Endod 2006;39:2-9.

18. Bernabe PF, Gomes-Filho JE, Rocha WC, et al. Histological evaluation of MTA as a root-end filling material. J Endod 2007;40:758-65.

19. Gomes-Filho JE, de Moraes Costa MT, Cintra LT, et al. Evaluation of rat alveolar bone response to Angelus MTA or experimental light-cured mineral trioxide aggregate using fluorochromes. J Endod 2011;37:250-4.

20. Schneider R, Holland GR, Chiego D Jr, et al. White mineral trioxide aggregate induces migration and proliferation of stem cells from the apical papilla. J Endod 2014; $40: 931-6$.

21. Holland R, Souza V, Nery MJ, et al. Reaction of rat connective tissue to implanted dentin filled with a white mineral trioxide aggregate. Braz Dent J 2002;13:23-6.
22. Camilleri J, Montesin F, Papaioannou S, et al. Biocompatibility of two commercial forms of mineral trioxide aggregate. Int Endod J 2004;37:699-704.

23. Gomes-Filho JE, Queiroz IO, Watanabe S, et al. Influence of diabetes mellitus on tissue response to MTA and its ability to stimulate mineralization. J Endod 2015;31:67-72.

24. Rodriguez-Iturbe B, Pons H, Quiroz Y, et al. Mycophenolate mofetil prevents saltsensitive hypertension resulting from angiotensin II exposure. Kidney Int 2001; 59:2222-32.

25. Bonato CF, do-Amaral CC, Belini L, et al. Hypertension favors the inflammatory process in rats with experimentally induced periodontitis. J Periodontal Res 2012;2:1-10.

26. Bills CE, Eisenberg H, Pallante SL. Complexes of organic acids with calcium phosphate: the von Kossa stain as a clue to the composition of bone mineral. Johns Hopkins Med J 1971;128:194-207.

27. Oldenbourg R. Polarized light microscopy: principles and practice. Cold Spring Harb Protoc 2013;2013.

28. Young EW, McCarron DA, Morris CD. Calcium regulating hormones in essential hypertension. Importance of gender. Am J Hypertens 1990;3 (8 Pt 2):161S-6.

29. Nakagami H, Morishita R. Hormones and osteoporosis update. Effect of angiotensin II on bone metabolism. Clin Calcium 2009;19:997-1002.

30. Oshima T, Young EW. Systemic and cellular calcium metabolism and hypertension. Semin Nephrol 1995;15:496-503.

31. Cintra LT, Bernabé PF, de Moraes IG, et al. Evaluation of subcutaneous and alveolar implantation surgical sites in the study of the biological properties of root-end filling endodontic materials. J Appl Oral Sci 2010;18:75-82.

32. De Batista PR, Palacios R, Martín A, et al. Toll-Like receptor 4 upregulation by angiotensin II contributes to hypertension and vascular dysfunction through reactive oxvgen species production. PLoS One 2014;9:e104020.

33. Holland R, de Souza V, Nery MJ, et al. Reaction of rat connective tissue to implanted dentin tubes filled with mineral trioxide aggregate or calcium hydroxide. J Endod 1999;25:161-6.

34. Matt GD, Thorpe JR, Strother JM, McClanahan SB. Comparative study of white and gray mineral trioxide aggregate (MTA) stimulating a one- or two-step apical barrier technique. J Endod 2004:30:876-9.

35. Li Q, Coleman NJ. The hydration chemistry of ProRoot MTA. Dent Mater J 2015; 34:458-65. 